\title{
VII - EARLY STAGES OF STELLAR EVOLUTION
}




\title{
THEORETICAL AND OBSERVATIONAL ASPECTS OF
}

\section{YOUNG STARS OF INTERMEDIATE MASS}

\author{
FRANCESCO PALLA \\ Osservatorio Astrofisico di Arcetri, \\ L.go E. Fermi, 5, I-50125 Firenze, Italy
}

\begin{abstract}
An account is given of the observational and theoretical properties that characterize young stars of intermediate-mass $\left(2 \leq M_{*} / M_{\odot} \leq 10\right)$, known as Herbig $\mathrm{Ae} / \mathrm{Be}$ stars. The mass range of these objects is an interesting one, since it involves the occurrence of complex phenomena associated with the transition from fully convective configurations, typical of $T$ Tauri stars, to radiatively stable objects, as in the case of massive stars. An overview of relevant observations testifying the variety of surface phenomena associated with the Herbig $\mathrm{Ae} / \mathrm{Be}$ stars is presented. Recent developments in the theory of the formation on intermediate-mass protostars will also be discussed.
\end{abstract}

Keywords : Protostellar and pre-main-sequence evolution

\section{Introduction}

There has been a relative lack of interest in recent years in the subject of protostars and pre-main-sequence (PMS) stars of intermediate mass $\left(2 \leq M_{*} / M_{\odot} \leq 10\right)$. Modern approach to the problem of star formation and PMS evolution has privileged the discussion of objects of solar and sub-solar mass, and a general, yet synthetic, account on the observational and theoretical foundations of the formation of sunlike stars has been given by Lada and Shu (1990). The reasons for the scarce attention paid to the stars under exam here are several.

Observationally, low mass stars outnumber more massive objects, a well known property epitomized by the Salpeter's law (1955) of the stellar distribution function. From the form of the initial mass function, we know that the probability of finding a star with $M_{*}=5 M_{\odot}$ is about 400 times smaller than that of the typical star, whose mass is $\approx 0.4 M_{\odot}$ according to current estimates (e.g. Scalo 1990). In addition to the statistical argument, the discovery that the dense and cold cores of molecular clouds are predominantly the sites of current low-mass star formation, has provided to the observers a powerfool tool for the detailed study of the physical conditions in individual regions that are relatively close to the Sun, and for comparative studies on a reasonably large sample of objects. At the same time, the observations have now reached enough resolution to test some specific predictions of the theoretical models so far developed, as convincingly illustrated in the contribution by N. Evans (this volume).

Theoretically, the reason for concentrating the largest effort on modelling lowmass star formation is easily explained. Low-mass protostars follow an evolution that is qualitatively different from that of their more massive counterparts. From a comparison of the relevant timescales, the accretion time onto the protostellar 
core $t_{a c c}=M_{*} / \dot{M}$, and the time needed to the core to reach internal equilibrium $t_{K H}=G M_{*}^{2} / R_{*} L_{*}$, we see that, unlike the former, the Kelvin-Helmoltz timescale is strongly dependent on mass. As long as $t_{a c c}<t_{K H}$, the protostar keeps accreting matter without changing dramatically its internal structure; but when $t_{a c c}>t_{K H}$ a transition occurs where the infall lags behind the gravitational contraction of the core : thus, the star will end its PMS phase of quasi-static contraction and join the main-sequence while still accreting. This transition is quite rapid and, due to the strong dependence of $t_{K H}$ on mass, interests only a rather narrow range of masses between $2 M_{\odot}$ and $3 M_{\odot}$. As a consequence, there should be no optically visible PMS stars above these masses, since the protostellar cores have already reached the conditions for hydrogen burning in the center and should quietly evolve as main-sequence stars. These qualitative analysis was soon confirmed by the first hydrodynamical calculations of the gravitational collapse of interstellar clouds (Larson 1972), and has remained unquestioned thereafter (cf. the discussion in the review article by Shu, Adams and Lizano 1987).

As a summary of the theoretical studies at the base of the classical protostellar and PMS theories for intermediate-mass objects, two results stand out :

1 - accreting protostars join the main-sequence at a mass $M_{*}=2-3 M_{\odot}$ (Larson 1972; Appenzeller 1980);

2 - PMS evolutionary tracks predict that intermediate-mass stars have fully radiative configurations (Iben 1965; Ezer and Cameron 1965).

These predictions must be confronted with two well established observational results, namely :

1 - PMS stars of intermediate-mass are known to exist : following Herbig's (1960) original suggestion about their nature, they are classified as Herbig Ae/Be stars (HAEBE);

2 - these stars show the same evidence of complex surface activities observed in $\mathrm{T}$ Tauri stars that, on the contrary, possess convective interiors.

Clearly, the classical theoretical models are insufficient to explain these two facts. It is the aim of this contribution to present an overview of the evolution of the field, in the light of recent results that help removing the serious inconsistencies between theory and observations. It is also hoped that the reader will be convinced that the subject of young intermediate-mass stars is a highly interesting one, sharing many, if not all, of the exciting theoretical and observational properties that have made the study of low-mass star formation so fashionable.

\section{Observational background}

There are about 60 known stars that satisfy the criteria suggested by Herbig to belong to the class of intermediate-mass PMS objects. This number is much smaller than that predicted by Herbig from a consideration of the relative PMS contraction time to the main-sequence lifetimes : accordingly, there should be several hundred contracting stars of large mass within $1 \mathrm{kpc}$ of the Sun. The large discrepancy is 
partly due to the fact that a systematic survey has not been carried out yet, and also that not all of them should be actually observable or fulfill all of Herbig's spectroscopic criteria. The latter were established entirely by analogy with the $\mathrm{T}$ Tausi stars, namely presence of emission lines in the spectra and association with regions of nebulosity and heavy obscuration.

The recognition that the stars proposed by Herbig (a sample of only 26 stars, originally) were indeed young stars still in the PMS phase came from the work of Strom et al. (1972), who determined the effective temperatures and surface gravities for some of them, and of Cohen and Kuhi (1979) who showed that their location in the HR diagram is well above the ZAMS. The most recent compilation of HAEBE is that of Finkenzeller and Mundt (1984) and includes 57 entries. Their catalogue has served as the basis for the detailed study of individual sources, and is slowly being expanded by new additions that mainly come from the recognition of new members selected. according to their far-infrared (FIR) colours (e.g. Hu et al. 1989). Figure 1 shows the HR diagram of the HAEBE stars, together with the region occupied by $T$ Tauri stars for comparison. The figure gives schematically the magnitude of the projected rotational velocities for the two samples, a property that will be commented upon below.

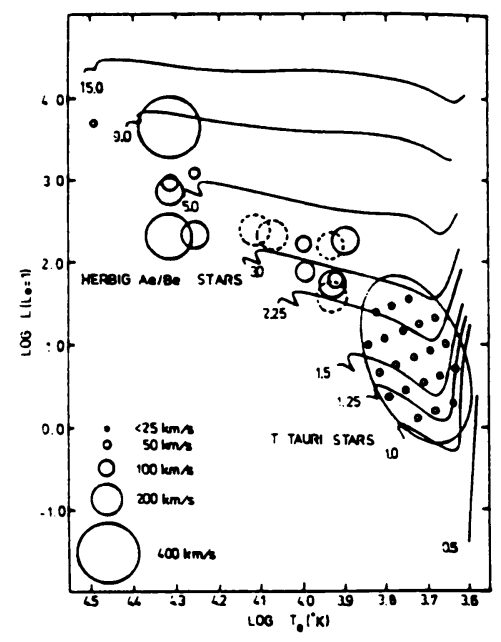

Fig. 1. Location of the HAEBE and T Tauri stars in the HR diagram, with the evolutionary tracks of Iben (1965). The magnitude of the projected rotational velocities $v$ sin $i$ is given by the size of the circles. (From Finkenzeller 1985).

The concentrated effort of several groups on few prototypes of the HAEBE class has resulted in the derivation of a coherent, although still incomplete, picture of their main properties. In the context of this review, it suffices to mention briefly the most important of them, but for a full account the interested reader is suggested to refer to the thorough summary given by Catala (1989). Schematically, HAEBE stars are characterized by : 
- extended chromospheres and strong stellar winds, as indicated by the high resolution profiles of several spectral lines ( $\mathrm{H} \alpha, \mathrm{Mg}$ II $\mathrm{h}$ and $\mathrm{k}, \mathrm{CaII} \mathrm{K}, \mathrm{HeI}$ and NaID, CIV etc.);

- significant variability and periodicity in the lines, interpreted in terms of rotational modulation (Praderie et al. 1986) or, alternatively, nonradial pulsations (Baade and Stahl 1989);

- strong UV depletion and IR excesses up to $100 \mu \mathrm{m}$, the latter indicative of the presence of warm circumstellar dust shells;

- molecular flows, highly collimated optical jets and Herbig-Haro objects, and maser emission;

- radio continuum emission.

The similarities of these phenomena with those commonly observed in $\mathrm{T}$ Tauri stars seems to indicate that star formation and early stellar evolution are all marked by the occurrence of the same phenomena over an extended mass interval. The major difference between HAEBE and $\mathrm{T}$ Tauri stars is in that the former rotate faster, typically with $v \sin i$ between 100 and $200 \mathrm{~km} \mathrm{~s}^{-1}$, and are depleted of slow rotators (cf. Fig. 1): the problem of the removal of the excess of angular momentum, althoug still present, is therefore less compelling than in the low-mass case.

There have been some interesting developments in the observations since Catala's review, and they will be discussed below.

\section{1. FIR EMISSION}

Berrilli et al. (1990) have presented the results of a search in the IRAS-PSC for FIR counterparts of the 57 HAEBE stars listed by Finkenzeller and Mundt. 35 sources are recognized to have most probable identification and their location in the $[60-25] /[25-12]$ colour-colour diagram is shown in panel a) of Figure 2. For comparison, panel $b$ ) shows the corresponding position of a sample of $T$ Tauri stars. Surprisingly, the two distributions are quite similar, despite the large difference in the luminosity output of the associated stars (a factor $10^{2}$ to $10^{4}$ ). Emission from circumstellar dust shells at temperatures varying between 90 and $170 \mathrm{~K}$ is responsible for the colours shown in the figure. Both classes of stars have FIR colours that are quite distinct from those of sources associated with highly luminous objects (ultracompact and more evolved HII regions), indicated by the small dots in fig. 2.

Berrilli et al. present the spectral energy distribution (SED) extending from the visual to $100 \mu \mathrm{m}$ for the 35 sources with FIR counterparts. Interestingly, the large majority of the sources ( 25 out of 37 ) have flat or very broad spectra, reminiscent of the T Tauris SEDs : the remaining 8 have spectra that decrease all the way to the longest wavelengths and that can be explained in terms of free-free emission. It would be interesting to see how the constraints set by the models developed to explain the infrared emission from $T$ Tauri stars apply also to the HAEBE stars. 


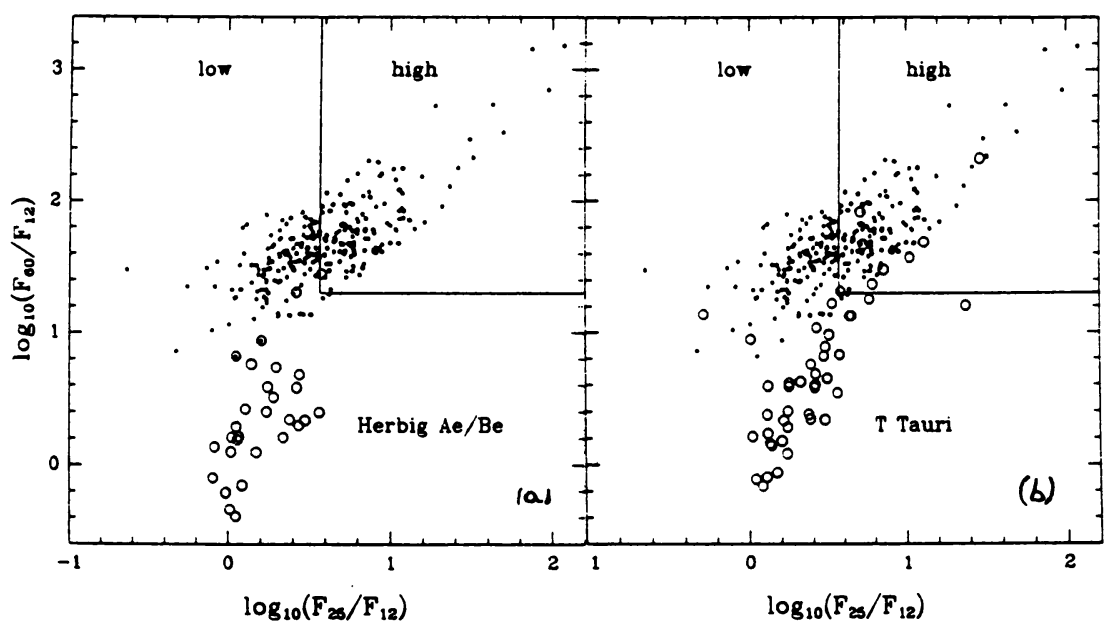

Fig. 2. Colour-colour diagrams of HAEBE (a) and T Tauri (b) stars. Small dots give the location of sources associated with early type stars. Sources within the box satisfy the colour criteria of Wood and Churchwell (1989) to discriminate ultracompact HII regions. (From Palla et al. 1990)

\section{2. RADIO CONTINUUM EMISSION}

Searches at radio wavelengths have been performed in the hope of gaining indirect evidence of the presence of surface magnetic fields, obviously required to help explaining the chromospheric activity unexpected in fully radiative stars. The most ambitious project is being carried out by Skinner, Brown, and Linsky who have recently published (Skinner et al. 1990) the initial results of a complete survey of the 57 known HAEBE stars done with the VLA. Continuum emission at 3.6 and 6 $\mathrm{cm}$ was detected at a rather low level (0.1-1.9 Jy) in 4 out 14 sources, namely : TY CrA, HD 200775, HD 259431, MWC 1080. In addition to these results, two other HAEBE stars have been detected : AB Aur by Güdel et al. (1989), and V645 Cyg by Curiel et al. (1989). As to the nature of the emission, unfortunately the results are not clear cut. In one case (HD 200775), the flux is consistent with free-free emission from a fully ionized, perhaps not spherically symmetric wind; while in another case (TY CrA) the spectrum has a negative slope, implying nonthermal emission. For the prototype of the class of HAEBE stars, AB Aur, the situation is more confusing, since the data at the shortest wavelength are consistent with thermal emission from a wind with characteristics derived from visible and UV data, but not those at $6 \mathrm{~cm}$. Obviously, any definite answer should await the completion of the survey.

\section{3. JETS AND $\mathrm{H}_{2} \mathrm{O}$ MASERS}

Ray et al. (1990) and Poetzl et al. (1989) have reported the discovery of HHlike objects in a large fraction (about $30 \%$ ) of young stars of intermediate to high 
luminosity. These are the first results of this kind, since previous searches have only dealt with sources of low luminosity (cf. Reipurth 1989). From the limited amount of information available at the present, it seems that the jets emanating from intermediate-mass PMS stars share the same characteristics (radial velocities, inferred mass loss rates, energy and momentum fluxes) of those associated with $\mathrm{T}$ Tauri stars.

Previous searches for $\mathrm{H}_{2} \mathrm{O}$ maser emission at $22.2 \mathrm{GHz}$ have all been characterized by a low detection rate. For example, the survey by Thum et al. (1981) toward 81 members of the Orion population yielded only 1 detection, $T$ Tauri. This prompted the authors to conclude that no $\mathrm{H}_{2} \mathrm{O}$ emission above the sensitivity limit of the survey $(\sim 1 \mathrm{Jy})$ is associated with stars in the mass range $1 \leq M_{*} / M_{\odot} \leq 10$. More recently, I have completed a survey of comparable sensitivity (Palla 1990) of 43 HAEBE stars and detected emission in 5 sources : 3 of them represent first time detections ( $\mathrm{T}$ Ori, $\mathrm{BD}+40^{\circ} 4124, \mathrm{Lk} \mathrm{H} \alpha 198$ ), while the other two were already known: V645 Cyg (Lada et al. 1981), and Lk $\mathrm{H} \alpha 234$ (Rodriguez and Canto 1983). Given the highly variable character of the maser emission, it can be concluded that, as in the case of more luminous stars, the occurrence of masers associated with intermediate-mass PMS is not an uncommon phenomenon (cf. also Rodriguez et al. 1987).

Overall, these new set of observations, together with those described in Catala's review, show with little doubt the complexity of the environment associated with the HAEBE stars.

\section{Theoretical overview}

\section{1. PROTOSTELLAR EVOLUTION}

From a theoretical point of view, intermediate-mass stars pose the challenging problem of studying in detail the transition from a state purely dominated by the accretion of interstellar gas onto the core to the gravitational contraction of the core itself, that can no longer remain fully convective and reverts to a radiatively stable configuration. However, the literature on the subject is scant. Since Larson (1972) classical paper on the simulation of the spherical accretion of protostellar cores, only the work of Yorke (1979) deals with the structure, evolution and appearance of stars in the mass interval of interest here. ${ }^{(1)}$ Yorke studied the hydrodynamical collapse of gas clouds of $3 \mathrm{M}_{\odot}$ and $10 \mathrm{M}_{\odot}$, respectively, from the onset of the gravitational instability to the end of the accretion phase, but did not follow the actual growth of the protostellar core, that was assumed to evolve in a parametric manner following Larson's results. Therefore, the latter remain the only self-consistent calculations available until now. It is important to remind that Larson's models, in

(1) For the sake of completeness, Shustov and Tutukov (1987) quote a paper by Tutukov and Chiefi (1985) on the evolution of accreting stars with mass $0.1 \leq$ $M_{*} / M_{\odot} \leq 6.6$, but unfortunately the detailed results are not available. 
addition to the usual limitation of assuming spherical symmetry, did not include the contribution in the energy equation of deuterium and hydrogen burning, thus missing, in the latter case, a precise estimate of the arrival of the contracting cores on the ZAMS. As for deuterium burning, Stahler (1988) has shown its fundamental role in establishing the main properties of low-mass protostars.

According to Larson's analysis, the formation and early evolution of intermediatemass stars is characterized by the occurrence of the following phenomena :

1 - for $M_{*} \geq 2 M_{\odot}$, the occurrence of a "luminosity wave" from the deep interiors of the core to the outer layers, accompained by a temporary and minor increase of the radius and of the total luminosity (about a factor of 2);

2 - for $M_{*} \sim 3 M_{\odot}$, the radiatively stable core goes through the entire PMS phase while still gaining mass from the infalling envelope, as shown in the theoretical HR diagram of Figure 3;

3 - for $M_{*} \geq 3 M_{\odot}$, the action of radiation pressure on the infalling grains should also be able to reverse the flow and halt the accretion.

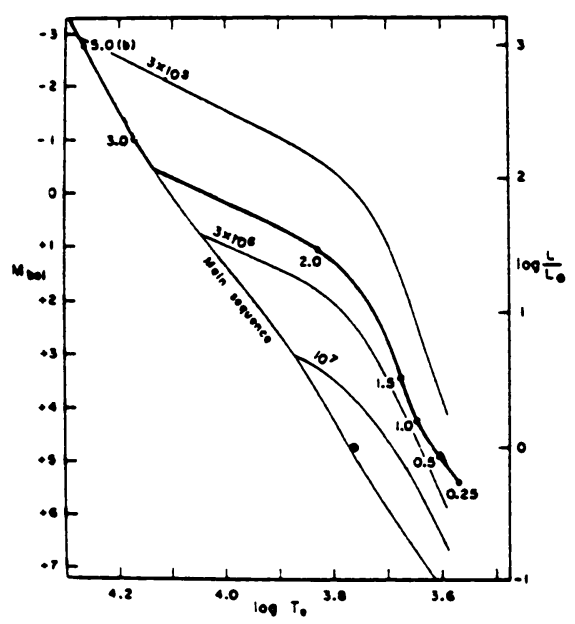

Fig. 3. The predicted locus (heavy curve) of newly formed stars in the HR diagrams according to Larson's calculations. Open circles mark the mass of the accreting protostellar core. Light curves are the isochrones from Iben and Talbot (1966).(Adapted from Larson 1972)

The occurrence of a luminosity wave inside the core has been reanalyzed by Stahler $(1989 \mathrm{~b})$, in the light of the results of modern protostellar theory. Stahler has shown that the luminosity increase due to the eruption of the wave at the surface of the core can be substantial, up to 30 times the initial luminosity for a star of $5 \mathrm{M}_{\odot}$, and suggests that this mechanism could be partly responsible for the 
occurence of the wild variations observed in PMS stars of comparable mass, such as the FU Ori objects.

As for the second aspect, the fact that the accreting cores reach the ZAMS at a rather low mass $\left(2-3 \mathrm{M}_{\odot}\right)$ is a consequence of having neglected deuterium burning, whose main effect is to establish a mass-radius relation for low-mass cores such that the radius increases proportionally with mass. We shall return at length on the importance of deuterium burning in more advanced stages of the protostellar evolution.

Finally, the estimate of the critical mass where radiation pressure effects become relevant has been revised upward, using better values of the dust opacity at visual wavelengths. It is found that radiation pressure takes over gravity when the luminosity to mass ratio satisfies the condition : $L_{*} / M_{*} \approx 700 L_{\odot} / M_{\odot}$ (Shu et al. 1987), a value reached by main-sequence stars more massive than about 7 $\mathrm{M}_{\odot}$ (see also the discussion in Nakano 1989). As we shall see below, protostars of comparable masses never attain such high values, so that the argument that the accretion phase might terminate due to radiation pressure is very weak. Given the amount of observational evidence of winds and energetic outflows associated with HAEBE stars, the latter seem to offer a more efficient mechanism.

\section{2. PRE-MAIN-SEQUENCE EVOLUTION}

The basic results found by Iben (1965) and Ezer and Cameron (1965) have remained unquestioned, despite the approximations in what concerns the initial conditions of the model calculations. A review of the various sources of uncertainties in the computation of the evolutionary tracks has been given by Mazzitelli (1989), but it is limited to PMS stars less massive than $1.2 \mathrm{M}_{\odot}$ and does not discuss the modifications in the initial conditions introduced by the results of the protostellar models. A discussion of this point can be found in Stahler (1989a). The main point is that using the so-called "Hayashi initial conditions", that require fully convective configurations, the stars are forced to have extremely high values of the radius at the beginning of the PMS evolution. Based on simple energetic arguments, Cameron (1962) obtained an initial value of the radius given by : $R_{P M S}^{i n i t} \approx 50 R_{\odot}\left(M_{*} / M_{\odot}\right)$. According to the results of the accretion phase, that should provide the correct initial conditions, the protostellar cores never attain such large radii, but remain a factor of $\sim 10$ below Cameron's estimate (Winkler and Newman 1980; Stahler, Shu and Taam 1980). However, this large discrepancy does not affect in a qualitative manner the evolution of low-mass PMS stars, since they satisfy the assumption about internal convection, driven by the action of deuterium burning in the center during the accretion phase. Indeed, the location of the theoretical birthline (Stahler 1983) and the position of the most luminous $T$ Tauri stars in the HR diagram accords well with the beginning of the evolutionary tracks in the Hayashi phase computed by Iben. On the contrary, more massive protostellar cores develop radiative interiors : thus, they will start the PMS phase strongly departing from the classical assumptions and the use of the evolutionary tracks to interpret the 
observed properties of intermediate-mass PMS stars will become less and less appropriate. We will now discuss the results of some recent calculations that can help to answer some of the fundamental questions raised in the Introduction.

\section{3. RECENT DEVELOPMENTS}

Palla and Stahler (1990a,b) have extended previous calculations of the spherical accretion of protostellar cores limited to $M_{\text {core }}=1 M_{\odot}$, to cover the entire mass interval up to the point where the cores reach the ZAMS. The novel feature of the evolution is the discovery of the onset of deuterium burning in a shell at the time of the transition to radiative stability. This shell burning alters the succession of events prior to the arrival onto the ZAMS in a dramatic way. Schematically, the entire burning process can be divided into 3 main stages :

Central D-burning: it represents the main property of the evolution of protostellar cores up to $M_{\text {core }} \sim 1 M_{\odot}$. The steady-state burning occurs near the center and keeps the star fully convective. The thermostatic nature of the burning ensures that the core radius grows steadily with mass.

The radiative barrier and the fading of central D-burning: as more matter is added to the core, the interior temperaure increases, the opacity drops, and the radiative luminosity becomes adequate in carrying away that produced by the steady-state burning of deuterium. Therefore, an internal zone will first become radiatively stable, so that the newly accreted deuterium can no longer be transported to the center of the star. This way, the whole region interior to the radiative barrier quickly burns the remaining deuterium and becomes radiatively stable.

The onset of $D$-shell burning:- as soon as the temperature of the elements outside the barrier reaches the critical value of $\sim 10^{6} \mathrm{~K}$, deuterium ignites in a shell maintaining convection in the outer layers. The luminosity of the burning shell rises quickly to attain its steady-state value, as illustrated in Figure 4. This energy source persists as the star accretes matter, but slowly retreats to the surface, due to the increased rate of energy released by the gravitational contraction of the inner parts. The situation is reminiscent of the "luminosity wave" discussed earlier, but in this case it has a completely different nature and its effects on the structure of the core become much more dramatic. It is found that, as the shell moves toward the surface, the outer layers expand considerably and the core doubles its size. This behavior can be appreciated by an inspection of Figure 5 that shows the run of the core radius vs. mass, obtained in the case of spherical accretion at a representative accretion rate of $10^{-5} M_{\odot} y r^{-1}$. This result is quite general and has a weak dependence on the details of the accretion process, whether from direct infall, as in the example presented here, or mediated by the presence of a circumstellar disk. The rapid increase in the core radius is followed by a slow decline, due to the increased strength of the gravitational pull as more matter is added onto the core. The major consequence of this behavior is that the interior temperature remains rather low, thus postponing the time of the onset of nuclear burning in the center. According to Fig. 5, hydrogen burning starts at a core mass $M_{\text {core }} \sim 6 M_{\odot}$, but the ZAMS is only 
reached at $M_{\text {core }} \sim 8 M_{\odot}$. In analogy with the low-mass protostars, the knowledge of the mass-radius relation allows one to derive the birthline of intermadiate-mass stars in the HR diagram, as shown by the heavy curve in Figure 6.

The agreement between the location of the birthline and the distribution of the observed HAEBE stars (the $T$ Tauri stars have been omitted for clarity) is remarkable : in accordance with the prediction, there is no optically visible PMS phase for $M_{*} \geq 9 M_{\odot}$. The comparison with the similar curve obtained by Larson, and shown in Fig. 3, underlines the crucial role that D-shell burning plays in setting the proper conditions in the accreting cores, and in reconciling the theoretical predictions with, at least a set of, the observations.

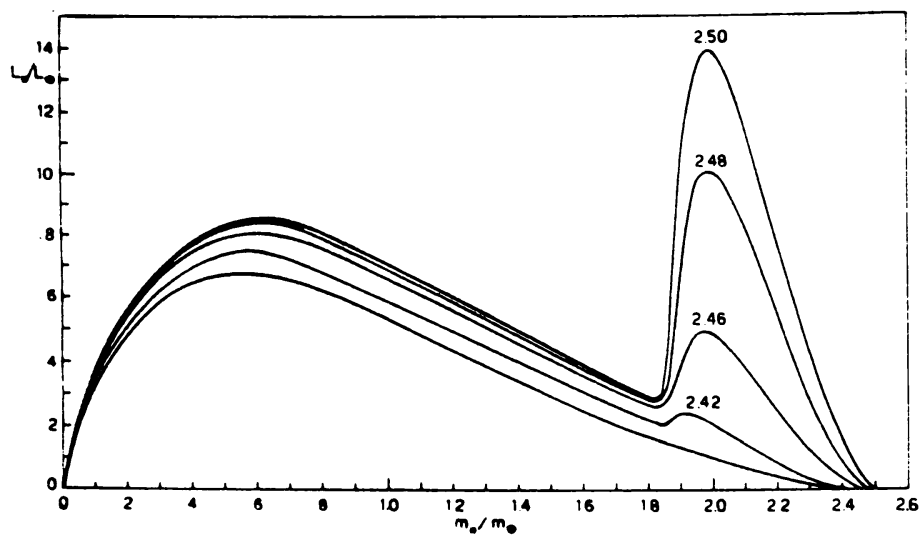

Fig. 4. The rise of the luminosity in the burning shell of an accreting protostar a. different epochs. Curves are labelled by the core mass. (From Palla and Stahler 1990b)

\section{Discussion}

The implications of the results presented in the previous section are numerous. First of all, given the position of the birthline vis à vis the evolutionary tracks of Iben, it is evident that the classical assumptions about intermediate-mass PMS stars must be revised. Not only the initial radii, set at the end of the accretion phase, are vastly different from those commonly assumed, but also the internal configuration of the stars presents a much more complicated structure. For example, a PMS star of $3 M_{\odot}$ will begin its evolution with a radiative core and a convective region extending all the way to the surface, due to the action of D-burning. The internal luminosity distribution will show a double peak, the off-center maximum due to the radiative luminosity and that at the position of the burning shell, and the ensuing nonhomologous contraction will not occur in a simply predictable manner.

Another implication of the birthline concerns the age estimate of the HAEBE stars, that in reality should be much younger than expected on the basis of the evolutionary tracks : the difference can be appreciable, up to even $50 \%$ of the 


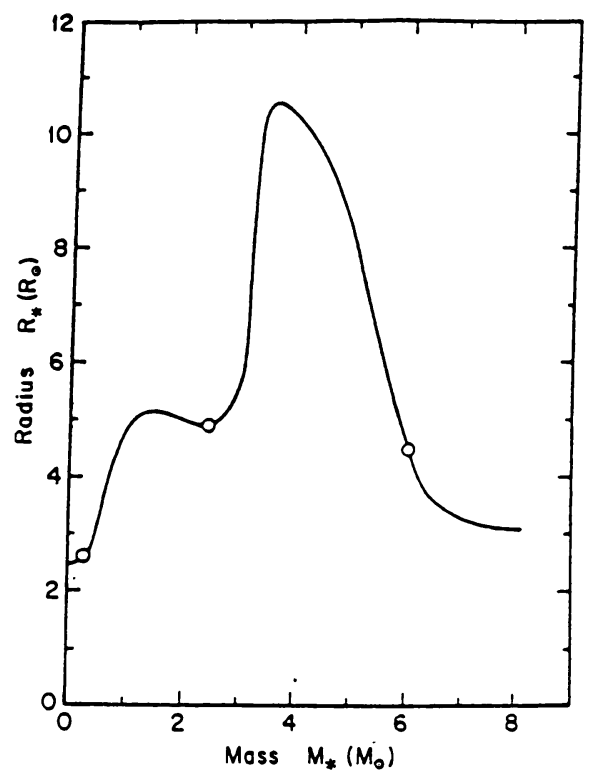

Fig. 5. The core mass-radius relation for accreting protostars. The various phases of the accretion phase and the main burning events are schematically indicated. (Adapted from Palla and Stahler 1990a)

traditional value. An indirect sign of their relative youth is given by the association with HH-like objects, molecular outflows, and masers previoulsy discussed. This in turn implies that the stars have been active for a shorter period of time, thus releasing some constraints on the mechanism(-s) responsible for the activity.

Finally, the role of D-burning in promoting surface convection in stars that were thought to have none, can indeed resolve the paradox brought about by the observations. Not only that, but the onset of nuclear reactions near the surface can also excite instabilities that will possibly develop (non)radial oscillations that could account for the observed rapid variability in the spectral lines. This possibility was alluded to by Herbig (1983), who noted that the PMS tracks of stars more massive than $2 M_{\odot}$ must cross the post-main-sequence instability strip. In this regard, it is important to remind the work of Toma (1972) who studied the radial pulsational instability of low-mass PMS stars due to central D-burning in fully convective configurations (see also the recent discussion of Gahm and Liseau 1988). The fact that intermediate-mass protostars possess radiative cores and D-burning shells makes a simple estimate of the predicted oscillation periods much more difficult : a re-analysis of the problem with more realistic.conditions is warranted.

Obviously, the questions and problems left to be answered are numerous. The few issues that have been stressed here reveal how the field is still in its infancy, compared to the more mature subject of low-mass star formation, where the role 


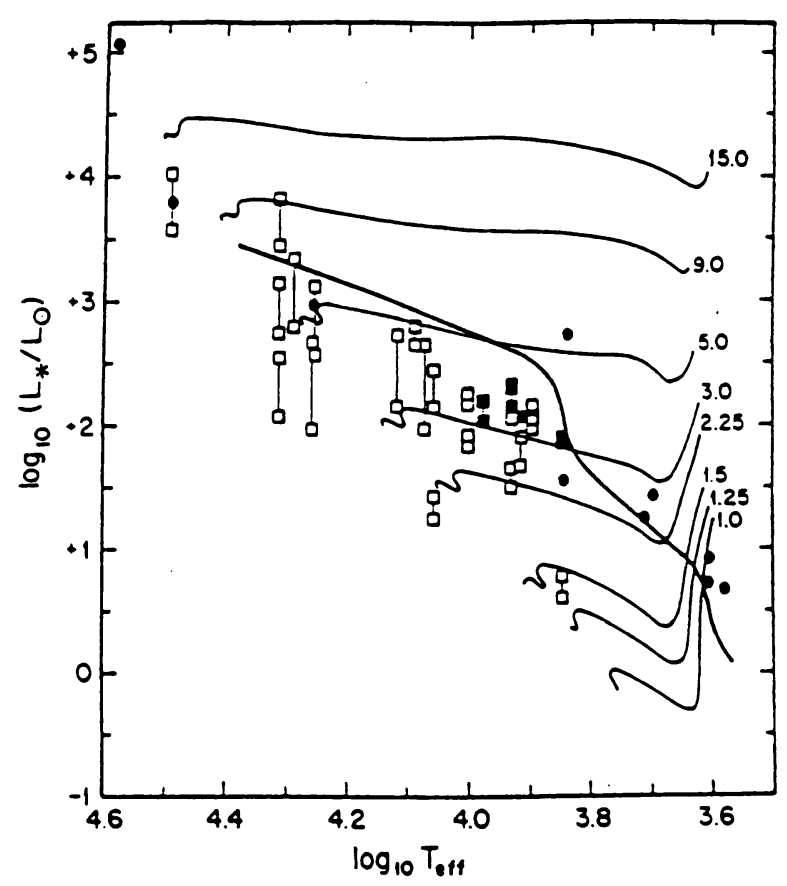

Fig. 6. The theoretical birthline (heavy curve) of intermediate-mass PMS stars. The squares are observed HAEBE stars with the uncertainty in the luminosity, due to different assumptions about the extinction (Finkenzeller and Mundt 1984). The filled circles are optically visible stars with CO outflows (Levreault 1988). The evolutionary tracks are from Iben (1965). (From Palla and Stahler 1990a)

of rotation and magnetic fields, the presence of discs etc., not discussed in this context, have been incorporated already in a more coherent way. On the observational side, the question of the occurrence of binaries among HAEBE stars should be attacked soon, as well as the study of these stars in the youngest galactic clusters to learn about possible evolutionary trends. On the theoretical side, much more refinement in the current models is demanded, and the calculation of updated PMS evolutionary tracks should have the highest priority, especially in the light of the wealth of observational material brought about by the study of the embedded star clusters in star forming regions.

\section{Acknowledgements}

It is a pleasure to thank the Organizing Committee, and in particular Edith Falgarone, for allocating ample time to present and discuss at the Meeting the main ideas collected in this contribution. All of the numerical calculations presented here 
come from a collaborative effort with Steve Stahler, an endeavor that started a long time ago. Finally, special thanks go to Claude Catala for his kind hospitality at the DESPA-Observatoire de Meudon and for sharing the excitment about Herbig stars.

\section{References}

Appenzeller, I. : 1980, in Star Formation, A. Maeder and L. Martinet eds., p.3.

Baade, D. and Stahl, O. : 1989, Astron. Astrophys., 209, 268.

Berrilli, F., Ceccarelli, C., Lorenzetti, D., Nisini, B., Saraceno, P. and Strafella, F. : 1990, Il Nuovo Cimento, 13C, 293.

Cameron, A.G.W.: 1962, Icarus, 1, 13.

Catala, C. : 1989, in Low Mass Star Formation and Pre-Main-Sequence Evolution, ESO Conf. and Work. Proc., Bo Reipurth ed., p.471.

Cohen, M., Kuhi, L.V. : 1979, Astrophys. J. Suppl., $41,743$.

Curiel, S., Rodriguez, L.F., Cantó, J., Bohigas, J., Roth, M. and Torrelles, J.M. : 1989, Astro. Lett. Comm., 27, 299.

Ezer, D. and Cameron, A.G.W. : 1965, Canadian J. Phys., 43, 1497.

Finkenzeller, U. : 1985, Astron. Astrophys., 151, 340.

Finkenzeller, U. and Mundt, R. : 1984, Astron. Astrophys. Suppl., 55, 109.

Gahm, G.F. and Liseau, R. : 1988, in Activity in Cool Star Envelopes, O. Havnes et al. eds., Kluwer Acad. Publ., p.99.

Güdel, M., Benz, A., Catala, C. and Praderie, F. : 1986, Astron. Astrophys., 217, L9.

Herbig, G.H. : 1960, Astrophys. J. Suppl., 4, 337.

Herbig, G.H. : 1983, in Birth and Infancy of Stars, Les Houches-Session XLI, R. Lucas, A. Omont and R. Stora eds., p.535.

Hu, J.Y., Thé, P.S. and de Winter, D. : 1989, Astron. Astrophys., 208, 213.

Iben, I. : 1965, Astrophys. J., 141, 993.

Iben, I and Talbot, R.J. : 1966, Astrophys. J., 144, 968.

Lada, C.J., Blitz, L., Reid, M.J., and MOran, J.M. : 1981, Astrophys. J., 243, 769.

Lada, C.J., and Shu, F.H. : 1990,Science, 248, 564.

Larson, R.B. : 1972, Monthly. Not. Roy. Astron. Soc., 157, 121.

Levreault, R.M. : 1988, Astrophys. J., 330, 910.

Mazzitelli, I. : 1989, in Low Mass Star Formation and Pre-Main-Sequence Evolution, ESO Conf. and Work. Proc., Bo Reipurth ed., p.433.

Nakano, T. : 1989, Astrophys. J., 345, 464.

Palla, F. : 1990, Astrophys. J. Lett., in press.

Palla, F. and Stahler, S.W. : 1990a, Astrophysical. J. Lett., 360, L47.

Palla, F. and Stahler, S.W. : 1990b, Astrophysical. J., in press.

Palla, F., Brand, J., Cesaroni, R., Comoretto, G. and Felli, M. : 1990, Astron. Astrophys, in press.

Poetzl, R., Mundt, R. and Ray, T.P. : 1989, Astronom. Astrophys., 224, L13.

Praderie, F., Simon, T., Catala, C. and Boesgard, R.M. : 1986, Astrophys. J., 303, 311.

Ray, T.P., Poetzl, R., Solf, J. and Mundt, R. : 1990, Astron. Astrophys., 357, L45.

Reipurth, B. : 1989, in Low Mass Star Formation and Pre-Main-Sequence Evolution, ESO Conf. and Work. Proc., Bo Reipurth ed., p.247.

Rodriguez, L.F. and Cantó, J. : 1983, Rev. Mex. Astron. Astrof., 8, 163.

Rodriguez, L.F., Haschick, A.D., Torrelles, J.M. and Myers, P.C. : 1987, Astron. Astrophys., 186, 319.

Salpeter, E.E. : 1955, Astrophys. J., 121, 161.

Scalo, J.M. : 1990, in Windows on Galaxies, A. Renzini, G. Fabbiano and J. Gallagher eds., Kluwer-Dordrecht, in press. 
Shu, F.H., Adams, F. and Lizano, S. : 1987, Ann. Rev. Astron. Astrophys., 25, 23.

Shustov, B.M. and Tutukov, A.V.: 1987, in Star Forming Regions, J. Jugaku and M. Peimbert eds., IAU Symposium 115, Reidel-Dordrecth, p.440.

Skinner, S.L., Brown, A. and Linsky, J.L. : 1990, Astrophys. J. Lett., 357, L39.

Stahler, S.W. : 1983, Astrophys. J., 274, 822.

Stahler, S.W. : 1988, Astrophys. J., 332, 804.

Stahler, S.W. : 1989a, Pubb. Astron. Soc. Pac., 100, 1474.

Stahler, S.W. : 1989b, Astrophys. J., 347, 950.

Stahler, S.W., Shu, F.H. and Taam, R.E. : 1980, Astrophys. J., 241, 637.

Strom, S.E., Strom, K.M., Yost, J., Carrasco, L., Grasdalen, G.L. : 1972, Astrophys. J., $173,353$.

Thum, C., Bertout, C., Downes, D. : 1981, Astron. Astrophys., 94, 80.

Toma, E. : 1972, Astron. Astrophys., 19, 76.

Tutukov, A.V. and Chief, A.: 1985, Nauchn. Inf., 58, 40.

Winkler, K.H. and Newman, M.J. : 1980, Astrophys. J., 236, 201.

Wood, D.O.S. and Churchwell, E. : 1989, Astrophys. J., 340, 265.

Yorke, H.W. : 1979, Astron. Astrophys., 80, 308. 